\begin{tabular}{|c|c|}
\hline Title & A llozyme variation and genetic differentiation in the loach Misgurnus anguillicaudatus \\
\hline Author(s) & Khan, Mukhlesur Rahman; A rai, Katsutoshi \\
\hline Citation & $\begin{array}{l}\text { Fisheries Science, 66(2), 211-222 } \\
\text { https://doi.org/10.1046j.1444.2906.2000.00037.x }\end{array}$ \\
\hline Issue Date & 2000 \\
\hline Doc URL & http:/hdl.handle.net/2115/35196 \\
\hline Rights & ○ 2000 公益社団法人日本水産学会; @ 2000 The Japanese Society of Fisheries Science \\
\hline Type & article \\
\hline File Information & arai-54.pdf \\
\hline
\end{tabular}

Instructions for use 


\title{
Allozyme variation and genetic differentiation in the loach Misgurnus anguillicaudatus
}

\author{
Mukhlesur Rahman KHAN AND Katsutoshi ARAI*,a
}

Faculty of Applied Biological Science, Hiroshima University, Higashi-hiroshima, Hiroshima 739-8528, Japan

\begin{abstract}
SUMMARY: Allozyme variation of diploid loach Misgurnus anguillicaudatus was examined in samples collected from 44 localities in Japan. Genetic differentiation was estimated based on allele frequencies of 12 enzyme loci. Nine loci $(36.9 \%)$ were polymorphic $(<0.95$ in major allele frequency) and average heterozygosities were unusually high $(H o=0.110 \pm 0.051, H e=0.131 \pm 0.052)$. The coefficient of gene differentiation ( $G s t=0.774$ ) suggested high genetic differentiation among populations. Based on the unweighted pair-group method using arithmetic averages (UPGMA) dendrogram, loach populations were grouped into six clusters. The population of Memanbetsu, Hokkaido (group 1) was remarkably different from others $(D=0.286)$. Populations distributed in Niigata, Nagano, Tochigi, Saitama, Chiba and Shizuoka Prefectures (group 6) were also genetically distinct from other loaches $(D=0.192)$. These results suggest genetic differentiation among groups 1,6 , and other loaches may be inter-subspecies level. Other loaches could be separated to four clusters (groups 2-5) at $D=$ 0.100-0.065. Genetic differentiation among them seems to be at local race level.
\end{abstract}

\section{KEY WORDS: allozyme, genetic distance, Misgurnus anguillicaudatus, population genetics.}

\section{INTRODUCTION}

The loach Misgurnus anguillicaudatus (Cobitidae, Cypriniformes) is widely distributed in Japan, Korea, Sakhalin, Taiwan and east coasts of Asian Continent from Amur River to North Vietnam. ${ }^{1}$ Although the taxonomic relationship among these loaches has not yet been clarified, presence of taxonomically or genetically distinct loaches has been suggested. Oliva and Hensel determined, on the basis of morphological observations, that the specimens from Yang-tse-kiang, China, belong to Misgurnus a. tungting Nichols, but those from Hyogo and Kagawa Prefectures, Japan, belong to M. a. anguillicaudatus Cantor. ${ }^{2}$ They also determined that the specimens from Nagasaki, Kyushu, and Kagawa Prefectures, Japan, belong to Misgurnus mizolepis elongatus Kimura. However, cytogenetic studies have shown that the loach M. anguillicaudatus from Hubei Province, China, is tetraploid with 100 chromosomes, ${ }^{3}$ but the loach in Japan is diploid with 50 chromosomes. ${ }^{4-6}$ The allelic

\footnotetext{
*Corresponding author: Tel: 81-138-40-5535. Fax: 81-138-40-5537. Email: araikt@fish.hokudai.ac.jp

aPresent address: Faculty of Fisheries, Hokkaido University, Hakodate 041-8611, Japan.

Received 2 April 1999.
}

differences were observed at the two diagnostic isozyme loci $\mathrm{LDH}-1 *$ and $\mathrm{MDH}-2 *$ between normal diploid loach $(2 n=50)$ and natural tetraploid loach $(4 n=100)$ which had been found in fish dealers.

In Japanese diploid loach, Kimura examined the genetic variation of samples from Aomori, Akita, Ibaraki, Aichi, Gifu and Shiga Prefectures by protein electrophoresis and concluded presence of two geographical races. ${ }^{8}$ Dong et al. strongly suggested sympatric distribution of the unknown loach species which has never been found as a species, together with $M$. anguillicaudatus in Torentsu river, Ibaraki Prefecture based on allozymes and morphological traits. ${ }^{9}$ Recently, Zhang and Arai reported 25 triploids in 1815 loaches collected from 35 localities in Japan and they also found the diploid loach, which laid unreduced diploid eggs, in the locality showing a relatively high frequency of triploids. ${ }^{10}$ They suggested that natural triploid loaches might have resulted from fertilization of such unreduced eggs. The occurrence of such unreduced diploid eggs has been reported in unisexual vertebrates with hybrid origin ${ }^{11}$ and interspecific hybrids. ${ }^{12}$ Thus, the appearance of such a unique diploid loach and natural triploid individuals suggest the presence of genetically distinct loaches and possible hybridization between them in nature.

As described above, previous genetic studies of this species are still fragmentary and inconclusive, but seem 
to be suggestive for the presence of genetically distinct loaches. In the present study, allozyme variation was surveyed among samples from 44 localities in Japan by starch-gel electrophoresis and then genetic differentiation was examined by the cluster analyses based on allele frequencies of gene loci examined.

\section{MATERIALS AND METHODS}

\section{Fish specimens}

Samples of the loach Misgurnus anguillicaudatus were collected from 44 different localities from Hokkaido, Honshu, Shikoku, and Kyushu Islands, Japan, from May. 1996 to October 1998 (Table 1). Loach samples from Nakamura (sample 43 in Table 1) were collected from the water way of a carp farm, and those from Hirokami (sample 18) were from a fish farm. Other samples were collected from rice fields, rivers, lakes, ponds, or artificial water ways. After confirmation of diploidy of each sample by flow-cytometry for DNA content of erythrocytes, ${ }^{13}$ muscle and liver tissues were taken and immediately frozen at $-80^{\circ} \mathrm{C}$, then stored until electrophoretic analysis. In samples collected, no mud loach $M$. mizolepis was found based on their external morphology.

\section{Electrophoresis}

The enzymes analyzed, E.C. numbers, abbreviation of enzymes and the buffer systems used for horizontal starch-gel electrophoresis are shown in Table 2. Electrophoresis was conducted using amine-citrate buffer (CA 6, CA 7) $)^{14}$ and tris-borate-citrate-lithium hydroxide buffer (TBCL) system. ${ }^{15}$ After electrophoresis, the gel slices (about $1 \mathrm{~mm}$ thickness) were histochemically stained for different enzymes as described in Allendorf et al. ${ }^{16}$ and Aebersold et al. ${ }^{17}$ with some modifications.

Loci were numbered consecutively from the anodal to the cathodal side. Thus, the most anodal one was designated ' 1 '. Gene nomenclature followed by Shaklee et al. ${ }^{18}$ The electrophoretic bands corresponding to multiple alleles at each locus were alphabetically named as $* a, * b$, $*_{c}$-.-- in the order of detection.

\section{Genetic analysis}

Allele frequencies were calculated directly from observed genotypes. The distribution of observed genotypes was compared with that expected, calculated from the Hardy-Weinberg equilibrium using a $\chi^{2}$ test.

When the most common (major) allele existed in a frequency less than 0.95 at a given locus, this locus was regarded as polymorphic. The mean proportions of heterozygous loci per individual, mean proportions of polymorphic loci per population and average number of alleles per locus were calculated so as to show the extent of genetic variability. ${ }^{19}$ Expected $\left(H_{e}\right)$ and observed average heterozygosity $\left(\mathrm{H}_{0}\right)$ were also calculated. ${ }^{20}$

The coefficient of gene differentiation $\left(G_{S T}\right)^{21}$ was calculated in order to estimate diversity between sample lots. Genetic distance values $(D)^{21}$ were calculated from allele frequencies for all possible pairs of sample lots. The analyses of allozyme data were performed using BIOSYS1 version $1.7 .^{22}$ Based on the $D$-values, a dendrogram was made using the unweighted pair-group method using arithmetic averages (UPGMA) method. ${ }^{23-25}$

\section{RESULTS}

\section{Allozyme variation and genotype}

The electrophoretic patterns of muscle and liver samples showed that the enzymes were controlled by 12 presumptive loci. Allele frequencies were calculated directly from observed genotypes at these loci in samples from 44 localities (Table 3 ).

Aspartate aminotransferase (AAT), esterase-D (EST$D)$, and tricerol-3-phosphate dehydrogenase (G3PDH) were dimeric enzymes and heterozygotes exhibited three banded pattern. Each enzyme was controlled by at least two loci. However, genotypes were presumed in AAT-1*, EST-D-1*, and G3PDH-1*, respectively. Creatine kinase (CK) was a monomeric enzyme controlled by at least two loci. The genotype was determined only in $\mathrm{CK}-2^{*}$.

Schematic representation of electrophoretic patterns of lactate dehydrogenase (LDH) is illustrated in Fig. 1. Lactate dehydrogenase was a tetrameric enzyme controlled by two loci: $L D H-1^{*}$ and $L D H-2^{*}$. Three alleles were involved in $\mathrm{LDH}-\mathrm{I}^{*}$ genotypes, while there were two alleles in $\mathrm{LDH}-2 *$.

Malate dehydrogenase $(\mathrm{MDH})$ was a dimeric enzyme controlled by four different loci: $\mathrm{MDH}-1^{*}, \mathrm{MDH}-2^{*}$, $\mathrm{MDH}-3^{*}$, and $\mathrm{MDH}-4 *$ (Fig. 1 ). The $\mathrm{MDH}-1 *$ locus was faintly expressed in both muscle and the liver, and reliable genotypes were difficult to read. In liver, genotypes of $\mathrm{MDH}-2 *$ were easily read because of the suppression of the $\mathrm{MDH}-3 *$ locus. Genotypes of $\mathrm{MDH}-3 *$ were presumed on the electrophoretic pattern of muscle samples. $\mathrm{MDH}-4^{*}$ was expressed both in the liver and muscle, and genotypes were presumed on the electrophoretic pattern.

Mannose phosphate isomerase (MPI) and phosphoglucomutase (PGM) were monomeric enzymes, controlled by single MPI* and PGM* locus, respectively. Three alleles were detected in both loci. Genotypes of PGM* locus are schematically shown in Fig. 1.

Superoxide dismutase (SOD) was a dimeric enzyme and controlled by a single SOD* locus which was strongly expressed in liver tissues. The SOD* locus was polymorphic and seven alleles were detected (Fig. 1). 
Table 1 Samples of the loach Misgurnus anguillicaudatus

\begin{tabular}{|c|c|c|c|c|}
\hline \multirow[t]{2}{*}{ Sample } & \multicolumn{2}{|r|}{ Locality } & \multirow[t]{2}{*}{ No. of fish } & \multirow[t]{2}{*}{ Date of collection } \\
\hline & Prefecture & Locality $^{1}$ & & \\
\hline 1 & Hokkaido & Memanbetsu T. [Hongou] & $20^{\circ}$ & June 98 \\
\hline 2 & & Higashikawa T. & 20 & August 96 \\
\hline 3 & & Iwamizawa C. & 46 & August 96 \\
\hline 4 & & Ebetsu C. (Sanaebetsu R.) & 14 & July 98 \\
\hline 5 & & Oono $\mathrm{T}$. & 33 & September 96 \\
\hline 6 & Akita & Kakunodate C. (Hinokinai R.) & 19 & July 98 \\
\hline 7 & Iwate & Morioka C. & 25 & September 96 \\
\hline 8 & & Oohasama T. (Hienuki R.) & 20 & October 96 \\
\hline 9 & & Towa T. (Sarugaishi R.) & 20 & November 96 \\
\hline 10 & & Kitakami C. (Kitakami R.) & 20 & November 96 . \\
\hline 11 & Miyagi & Naruko T. (Kawatabi P.) & 20 & June 98 \\
\hline 12 & & Shiwahime T. (Izunuma L.) & 20 & October 96 \\
\hline 13 & Fukushima & Tadami T. & 20 & October 96 \\
\hline 14 & Yamagata & Kushibiki T. (Itai R.) & 36 & September 96 \\
\hline 15 & Niigata & Maki T. & 20 & October 96 \\
\hline 16 & & Yahiko V. [Murayama] & 20 & June 97 \\
\hline 17 & & Nakanoshima T. [Nakanoshima] & 20 & July 97 \\
\hline 18 & & Hirokami V. & 12 & June 96 \\
\hline 19 & Tochigi & Nasu T. (Naka R.) & 40 & December 97 \\
\hline 20 & & Nikko C. (Naka R.) & 10 & August 98 \\
\hline 21 & Saitama & Hanyu C. (Kasai W.) & 17 & June 98 \\
\hline 22 & Chiba . & Futtsu T. (Iwase R.) & 20 & August 98 \\
\hline 23 & Nagano & Ueda C. (Urano R.) & 15 & July 98 \\
\hline 24 & Shizuoka & Inasa $\mathrm{T}$. & 19 & December 96 \\
\hline 25 & Mie & Tamaki T. [Iwade] & 20 & November 97 \\
\hline 26 & & Ise C. (Yokowa R.) & 20 & December 97 \\
\hline 27 & Shiga & Makino T. (Shourai R.) & 20 & August 96 \\
\hline 28 & & Imazu T. [Hamabun] & 20 & October 98 \\
\hline 29 & Fukui & Obama C. (Eko R.) & 20 & July 98 \\
\hline 30 & Gifu & Hashima C. [Masakicho, Ooura] & 20 & August 98 \\
\hline $31^{2}$ & Kyoto & Mineyama T. (A) [Masutome] & 20 & June 98 \\
\hline $32^{2}$ & & Mineyama T. (B) [Goka] & 20 & June 98 \\
\hline 33 & Tottori & Tomari V. [Ishiwaki] & 20 & June 98 \\
\hline 34 & Shimane & Izumo C. & 13 & November 97 \\
\hline 35 & Hiroshima & Higashi-hiroshima C. & 36 & October 97 \\
\hline 36 & & Hiroshima C. (Misasa R.) & 12 & October 97 \\
\hline 37 & Yamaguchi & Toyoura T. (Kawadana R.) & 37 & September 97 \\
\hline 38 & & Yuya T. (Asai R.) & 20 & June 98 \\
\hline 39 & Tokushima & Hiwasa T. & 20 & November 96 \\
\hline $40^{3}$ & Ehime & Uwa T. (A) [Kaminaru] & 20 & June 98 \\
\hline $41^{3}$ & & Uwa T. (B) [Kaida] & 20 & June 98 \\
\hline 42 & & Mima T. [Kitamasuho, Korenobu] & 12 & June 98 \\
\hline 43 & Kochi & Nakamura C. & 12 & August 96 \\
\hline 44 & Miyazaki & Saito C. & 15 & September 98 \\
\hline & & Total & 923 & \\
\hline
\end{tabular}

\footnotetext{
${ }^{1}$ Parentheses indicate name of river $(R)$, lake $(L)$, pond $(P)$, or water way $(W)$. Other samples were collected from rice fields or fish farm (samples
} 18 and 44 ). Bracket indicates name of a section in city (C), town (T), or village (V).

${ }^{2}$ Collected in different sections in the same town. Distance between the two places was very close (about $5 \mathrm{~km}$ ).

${ }^{3}$ Collected in different sections in the same town. Distance between the two places was very close (about $2 \mathrm{~km}$ ).

The $\chi^{2}$ test was made in all the cases of polymorphic loci between observed and expected genotypes, based on Hardy-Weinberg equilibrium. However, the test was not effective in many cases in which the expected values were $<5$.

\section{Genetic variability}

The mean proportion of heterozygous loci per individual . was $27.4 \pm 14.5$ (mean $\pm S D$ )\% from the average of 44 sample lots and ranged from $5.6 \%$ (sample 35 ) to $75.0 \%$ 
Table 2 Enzymes examined and electrophoretic buffers used

\begin{tabular}{lllll}
\hline Enzyme & Abbreviation & E.C. no. & Tissue $^{1}$ & Buffer system \\
\hline Aspartate aminotransferase & AAT & 2.6 .1 .1 & Muscle & CA 6 $6^{1}$ \\
Creatine kinase & CK & 2.7 .3 .2 & Muscle & TBCL $^{2}$ \\
Esterase-D & EST-D & $3.1 .1 .-$ & Muscle & CA 7 7 \\
Glycerol-3-phosphate dehydrogenase & G3PDH & 1.1 .1 .8 & Muscle & CA 7 \\
Lactate dehydrogenase & LDH & 1.1 .1 .27 & Muscle & CA.6 \\
Malate dehydrogenase & MDH & 1.1 .1 .37 & Muscle/liver & CA 6 \\
Mannose phosphate isomerase & MPI & 5.3 .1 .8 & Muscle & TBCL \\
Phosphoglucomutase & PGM & 2.7 .5 .1 & Muscle & CA 6 \\
Superoxide dismutase & SOD & 1.51 .1 .1 & Muscle/liver & TBCL
\end{tabular}

${ }^{1} \mathrm{CA}$ 6, amine-citrate buffer ( $\left.\mathrm{pH} 6.0\right) ;{ }^{13}{ }^{2} \mathrm{TBCL}$, Tris-borate-citrate-lithium hydroxide buffer ${ }^{14}{ }^{3} \mathrm{CA} 7$, amine-citrate buffer $(\mathrm{pH} 7.0) .{ }^{13}$
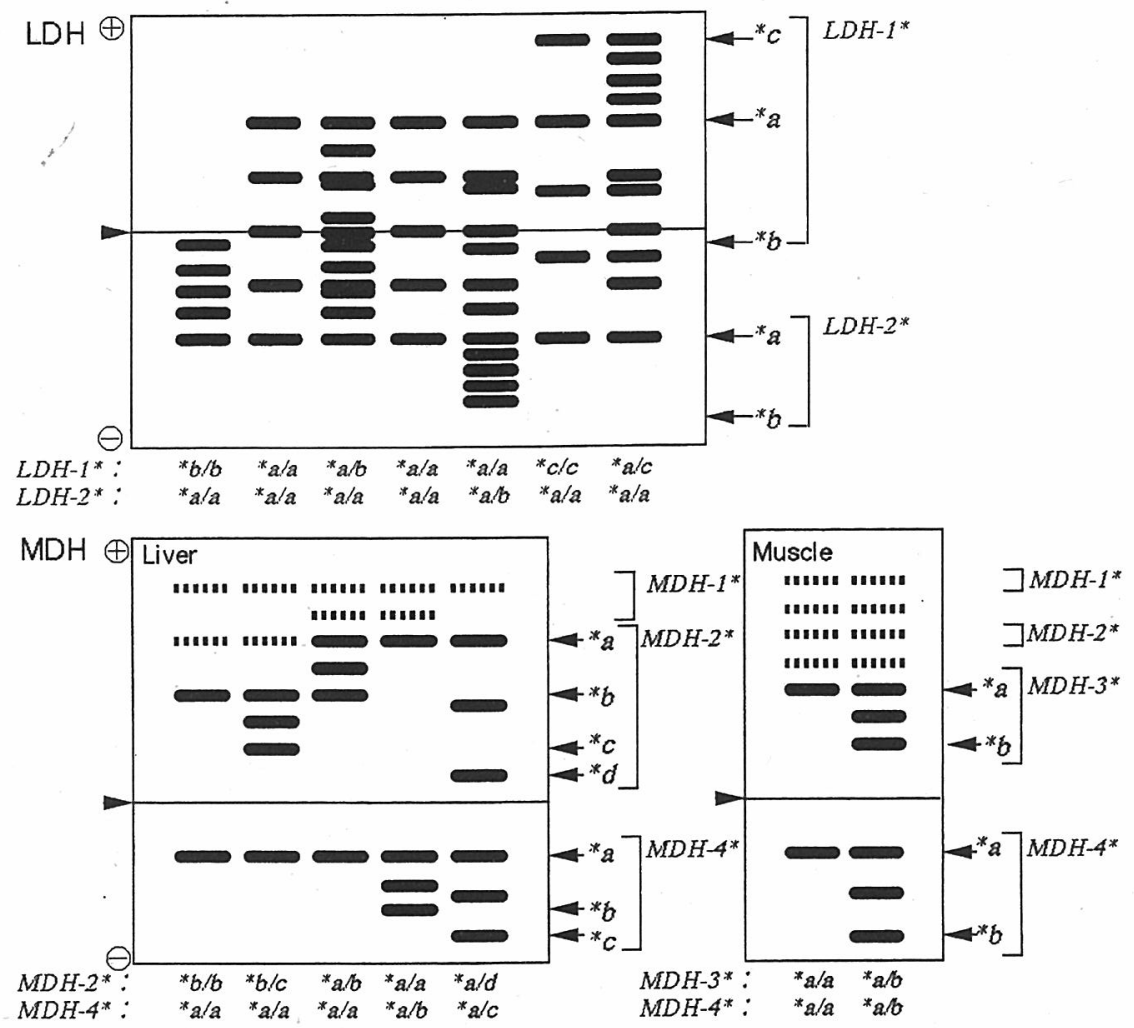

$M D H-4^{*}: \quad *_{a / a} *_{a / a} \quad *_{a / a} *_{a / b} *_{a / c} \quad M D H-4^{*}: \quad *_{a / a} *_{a / b}$

PGM

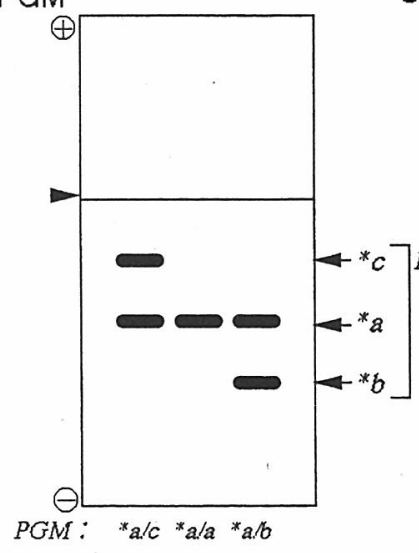

SOD
Fig. 1 Schematic representation of electrophoretic patterns in lactate dehydrogenase (LDH), malate dehydrogenase (MDH), phosphoglucomutase (PGM) and superoxide dismutase (SOD) of the loach Misgurnus anguillicaudatus. Genotypes of given locus were shown in the bottom of each illustration. Arrows indicate alleles of given locus. Arrowheads denote the origin of electrophoresis. 
Table 3 Allele frequencies at 12 presumptive loci of the loach Misgurnus anguillicaudatus

\begin{tabular}{|c|c|c|c|c|c|c|c|c|c|c|c|c|c|c|c|c|c|c|c|}
\hline \multirow[t]{2}{*}{ Sample } & \multicolumn{4}{|c|}{ AAT-1* } & \multicolumn{2}{|c|}{$C K-2^{*}$} & \multicolumn{4}{|c|}{ EST-D-I* } & \multicolumn{4}{|c|}{ G3PDH-I* } & \multicolumn{3}{|c|}{ LDH-I* } & \multicolumn{2}{|c|}{$\mathrm{LDH}-2 *$} \\
\hline & $* a$ & $* b$ & $*_{c}$ & $* d$ & $* a$ & $* b$ & $* a$ & $* b$ & ${ }^{*} c$ & $* d$ & $*_{a}$ & $* b$ & $*_{c}$ & $* d$ & $* a$ & $* b$ & ${ }^{*} c$ & $* a$ & $* b$ \\
\hline 1 & 0.650 & 0.350 & - & - & 1.000 & - & 0.900 & 0.100 & - & - & - & 0.550 & 0.450 & - & 1.000 & - & - & 1.000 & - \\
\hline 2 & 1.000 & - & - & - & 1.000 & - & 1.000 & - & - & - & - & 0.925 & 0.075 & - & 1.000 & - & - & 1.000 & - \\
\hline 3 & 1.000 & - & - & - & 1.000 & - & 0.794 & 0.184 & 0.022 & - & 0.033 & 0.771 & 0.196 & - & 1.000 & - & - & 1.000 & - \\
\hline 4 & 1.000 & - & - & - & 1.000 & - & 0.786 & 0.214 & - & - & 0.143 & 0.821 & 0.036 & - & 1.000 & - & - & 1.000 & - \\
\hline 5 & 1.000 & - & - & - & 1.000 & - & 1.000 & - & - & - & - & 0.878 & 0.092 & 0.030 & 1.000 & - & - & 1.000 & - \\
\hline 6 & 0.921 & 0.079 & - & - & 1.000 & - & 0.974 & 0.026 & - & - & 0.026 & 0.974 & - & - & 1.000 & - & - & 1.000 & - \\
\hline 7 & 1.000 & - & - & - & 1.000 & - & 0.980 & - & - & 0.020 & 0.020 & 0.840 & 0.140 & - & 1.000 & - & - & 1.000 & - \\
\hline 8 & 0.975 & 0.025 & - & - & 1.000 & - & 1.000 & - & - & - & 0.150 & 0.725 & 0.125 & - & 1.000 & - & - & 1.000 & - \\
\hline 9 & 1.000 & - & - & - & 1.000 & - & 0.850 & 0.150 & - & - & 0.132 & 0.842 & 0.026 & - & 1.000 & - & - & 1.000 & - \\
\hline 10 & 0.975 & 0.025 & - & - & 1.000 & - & 1.000 & - & - & - & 0.050 & 0.925 & 0.025 & - & 1.000 & - & - & 1.000 & - \\
\hline 11 & 1.000 & - & - & - & 1.000 & - & 1.000 & - & - & - & 0.050 & 0.475 & 0.475 & - & 1.000 & - & - & 1.000 & - \\
\hline 12 & 0.975 & 0.025 & - & - & 1.000 & - & 1.000 & - & - & - & - & 0.975 & 0.025 & - & 1.000 & - & - & 1.000 & - \\
\hline 13 & 1.000 & - & - & - & 1.000 & - & 0.925 & 0.075 & - & - & - & 0.925 & 0.075 & - & 1.000 & - & - & 1.000 & - \\
\hline 14 & 1.000 & - & - & - & 1.000 & - & 0.805 & 0.181 & 0.014 & - & - & 0.694 & 0.306 & - & 1.000 & - & - & 1.000 & - \\
\hline 15 & 0.900 & 0.025 & 0.075 & - & 1.000 & - & 0.925 & 0.075 & - & - & 0.025 & 0.775 & 0.200 & - & 0.125 & 0.875 & - & 1.000 & - \\
\hline 16 & 0.950 & 0.025 & 0.025 & - & 1.000 & - & 0.950 & 0.050 & - & - & 0.075 & 0.900 & 0.025 & - & 0.025 & 0.975 & - & 1.000 & - \\
\hline 17 & 0.875 & 0.025 & 0.100 & - & 1.000 & - & 0.875 & 0.125 & - & - & - & 0.900 & 0.100 & - & 0.225 & 0.775 & - & 1.000 & - \\
\hline 18 & 0.833 & 0.167 & - & - & 1.000 & - & 0.792 & 0.208 & - & - & 0.083 & 0.667 & 0.250 & - & 1.000 & - & - & 1.000 & - \\
\hline 19 & 0.846 & 0.103 & 0.051 & - & 1.000 & - & 0.850 & 0.150 & - & - & 0.012 & 0.863 & 0.125 & - & 0.213 & 0.787 & - & 1.000 & - \\
\hline 20 & 0.813 & 0.062 & 0.125 & - & 1.000 & - & 1.000 & - & - & - & - & 1.000 & - & - & - & 1.000 & - & 1.000 & - \\
\hline 21 & 0.909 & - & 0.091 & - & 1.000 & - & 1.000 & - & - & - & 0.059 & 0.912 & 0.029 & - & 0.059 & 0.941 & - & 1.000 & - \\
\hline 22 & 0.944 & 0.028 & 0.028 & - & 1.000 & - & 0.875 & 0.125 & - & - & - & 0.975 & 0.025 & - & 0.150 & 0.850 & - & 1.000 & - \\
\hline 23 & 0.900 & 0.033 & 0.067 & - & 1.000 & - & 0.866 & - & 0.134 & - & 0.167 & 0.767 & 0.066 & - & 0.067 & 0.933 & - & 1.000 & - \\
\hline 24 & 0.737 & 0.026 & 0.237 & - & 1.000 & - & 0.921 & 0.053 & 0.026 & - & 0.211 & 0.763 & 0.026 & - & - & 1.000 & - & . 1.000 & - \\
\hline 25 & 0.900 & 0.100 & - & - & 1.000 & - & 1.000 & - & - & - & 0.025 & 0.975 & - & - & 1.000 & - & - & 1.000 & - \\
\hline 26 & 1.000 & - & - & - & 1.000 & - & 0.975 & 0.025 & - & - & - & 0.975 & 0.025 & - & 1.000 & - & - & 1.000 & - \\
\hline 27 & 1.000 & - & - & - & 0.975 & 0.025 & 0.933 & 0.067 & - & - & 0.125 & 0.875 & - & - & 1.000 & - & - & 1.000 & - \\
\hline 28 & 1.000 & - & - & - & 1.000 & - & 0.925 & - & 0.075 & - & 0.025 & 0.925 & 0.050 & - & 1.000 & - & - & 1.000 & - \\
\hline 29 & 0.900 & - & - & 0.100 & 0.975 & 0.025 & 0.775 & 0.225 & - & - & 0.075 & 0.800 & 0.125 & - & 1.000 & - & - & 1.000 & - \\
\hline 30 & 0.975 & 0.025 & - & - & 1.000 & - & 0.950 & 0.050 & - & - & - & 1.000 & - & - & 0.900 & 0.100 & - & 0.950 & 0.050 \\
\hline 31 & 1.000 & - & - & - & 1.000 & - & 1.000 & - & - & - & 0.125 & 0.850 & 0.025 & - & 1.000 & - & - & 1.000 & - \\
\hline 32 & 1.000 & - & - & - & 1.000 & - & 1.000 & - & - & - & - & 1.000 & - & - & 0.975 & 0.025 & - & 0.975 & 0.025 \\
\hline 33 & 1.000 & - & - & - & 1.000 & - & 1.000 & - & - & - & 0.150 & 0.625 & 0.050 & 0.175 & 1.000 & - & - & 1.000 & - \\
\hline 34 & 0.769 & 0.213 & - & 0.018 & 1.000 & - & 1.000 & - & - & - & 0.038 & 0.962 & - & - & 1.000 & - & - & 1.000 & - \\
\hline 35 & 1.000 & - & - & - & 1.000 & - & 1.000 & - & - & - & - & 0.750 & 0.250 & - & 0.778 & - & 0.222 & 1.000 & - \\
\hline 36 & 1.000 & - & - & - & 1.000 & - & 1.000 & - & - & - & - & 0.792 & 0.208 & - & 1.000 & - & - & 1.000 & - \\
\hline 37 & 0.959 & 0.014 & 0.027 & - & 1.000 & - & 0.892 & 0.095 & 0.013 & - & - & 0.946 & 0.054 & - & 1.000 & - & - & 1.000 & - \\
\hline 38 & 1.000 & - & - & - & 1.000 & - & 0.850 & 0.150 & - & - & - & 1.000 & - & - & 1.000 & - & - & 1.000 & - \\
\hline 39 & 1.000 & - & - & - & 0.900 & 0.100 & 1.000 & - & - & - & 0.150 & 0.850 & - & - & 1.000 & - & - & 1.000 & - \\
\hline 40 & 1.000 & - & - & - & 1.000 & - & 0.400 & 0.600 & - & - & - & 1.000 & - & - & 1.000 & - & - & 1.000 & - \\
\hline 41 & 1.000 & - & - & - & 1.000 & - & 0.950 & 0.050 & - & - & - & 1.000 & - & - & 1.000 & - & - & 1.000 & - \\
\hline 42 & 1.000 & - & - & - & 1.000 & - & 0.708 & 0.292 & - & - & - & 1.000 & - & - & 1.000 & - & - & 1.000 & - \\
\hline 43 & 0.917 & 0.083 & - & - & 1.000 & - & 0.833 & 0.167 & - & - & 0.042 & 0.916 & 0.042 & - & - & 1.000 & - & 1.000 & - \\
\hline $44 \ldots$ & 0.933 & 0.067 & - & - & 1.000 & - & 1.000 & - & - & - & 0.267 & 0.700 & 0.033 & - & 0.167 & 0.833 & - & 1.000 & - \\
\hline
\end{tabular}


Table 3 (continued)

\begin{tabular}{|c|c|c|c|c|c|c|c|c|c|c|c|c|c|c|c|c|c|c|c|c|c|c|}
\hline \multirow[t]{2}{*}{ Sample } & \multicolumn{4}{|c|}{$M D H-2 *$} & \multicolumn{2}{|c|}{$\mathrm{MDH}-3^{*}$} & \multicolumn{3}{|c|}{$\mathrm{MDH}-4^{*}$} & \multicolumn{3}{|c|}{ MPI* } & \multicolumn{3}{|c|}{ PGM* } & \multicolumn{7}{|c|}{ SOD* } \\
\hline & $* a$ & $* b$ & $*_{C}$ & $* d$ & $* a$ & $* b$ & $* a$ & $* b$ & $*_{c}$ & $* a$ & $* b$ & $*_{c}$ & $* a$ & $* b$ & $*_{c}$ & $* a$ & $* b$ & $*^{*} c$ & $* d$ & $*_{e}$ & $*_{f}$ & $* g$ \\
\hline 1 & - & 0.925 & 0.075 & - & 1.000 & - & 1.000 & - & - & 0.675 & 0.325 & - & 0.925 & 0.050 & 0.025 & 0.100 & 0.775 & - & - & - & 0.125 & - \\
\hline 2 & 1.000 & - & - & - & 1.000 & - & 1.000 & - & - & 1.000 & - & - & 1.000 & - & - & 0.350 & 0.450 & - & 0.200 & - & - & - \\
\hline 3 & 1.000 & - & - & - & 1.000 & - & 1.000 & - & - & 1.000 & - & - & 1.000 & - & - & 0.462 & 0.313 & 0.038 & 0.112 & 0.075 & - & - \\
\hline 4 & 1.000 & - & - & - & 1.000 & - & 1.000 & - & - & 1.000 & - & - & 0.964 & - & 0.036 & 0.571 & 0.357 & - & 0.036 & 0.036 & - & - \\
\hline 5 & 1.000 & - & - & - & 1.000 & - & 0.984 & 0.016 & - & 1.000 & - & - & 0.969 & 0.031 & - & 0.625 & 0.375 & - & - & - & - & - \\
\hline 6 & 0.975 & 0.025 & - & - & 1.000 & - & 0.948 & 0.026 & 0.026 & 0.974 & 0.026 & - & 0.921 & 0.079 & - & 0.606 & 0.342 & - & 0.026 & - & - & 0.026 \\
\hline 7 & 1.000 & - & - & - & 1.000 & - & 1.000 & - & - & 1.000 & - & - & 1.000 & - & - & 0.380 & 0.620 & - & - & - & - & - \\
\hline 8 & 1.000 & - & - & - & 1.000 & - & 1.000 & - & - & 1.000 & - & - & 1.000 & - & - & 0.300 & 0.700 & - & - & - & - & - \\
\hline 9 & 0.525 & 0.475 & - & - & 1.000 & - & 1.000 & - & - & 0.650 & 0.350 & - & 0.925 & 0.075 & - & 0.375 & 0.575 & 0.050 & - & - & - & - \\
\hline 10 & 0.975 & 0.025 & - & - & 1.000 & - & 1.000 & - & - & 1.000 & - & - & 1.000 & - & - & 0.450 & 0.550 & - & - & - & - & - \\
\hline 11 & 1.000 & - & - & - & 1.000 & - & 1.000 & - & - & 1.000 & - & - & 1.000 & - & - & 0.750 & 0.250 & - & - & - & - & - \\
\hline 12 & 1.000 & - & - & - & 1.000 & -' & 1.000 & - & - & 1.000 & - & - & 1.000 & - & - & 0.600 & 0.400 & - & $\cdot-$ & - & - & - \\
\hline 13 & 1.000 & - & - & - & 1.000 & - & 1.000 & - & - & 1.000 & - & - & 0.950 & 0.050 & - & 0.625 & 0.350 & - & 0.025 & - & - & - \\
\hline 14 & 1.000 & - & - & - & 1.000 & - & 1.000 & - & - & 1.000 & - & - & 0.736 & 0.264 & - & 0.444 & 0.556 & - & - & - & - & - \\
\hline 15 & - & 1.000 & - & - & 1.000 & - & 1.000 & - & - & 0.725 & 0.275 & - & 0.750 & 0.150 & 0.100 & 0.125 & 0.450 & 0.175 & 0.225 & - & 0.025 & - \\
\hline 16 & - & 1.000 & - & - & 0.975 & 0.025 & 1.000 & - & - & 0.625 & 0.375 & - & 0.750 & 0.100 & 0.150 & 0.125 & 0.700 & 0.125 & 0.050 & - & - & - \\
\hline 17 & - & 1.000 & - & - & 0.975 & 0.025 & 1.000 & - & - & 0.775 & 0.225 & - & 0.750 & 0.075 & 0.175 & 0.150 & 0.350 & 0.325 & 0.150 & - & 0.025 & - \\
\hline 18 & 0.916 & 0.042 & - & 0.042 & 0.958 & 0.042 & 0.958 & 0.042 & - & 0.708 & 0.292 & - & 0.625 & 0.375 & - & - & 0.917 & 0.042 & - & 0.041 & - & - \\
\hline 19 & 0.050 & 0.950 & - & - & 1.000 & - & 1.000 & - & - & 0.700 & 0.300 & - & 0.825 & 0.062 & 0.113 & 0.125 & 0.563 & 0.150 & 0.050 & 0.112 & - & - \\
\hline 20 & - & 1.000 & - & - & 1.000 & - & 1.000 & - & - & 0.700 & 0.250 & 0.050 & 0.800 & 0.100 & 0.100 & 0.050 & 0.550 & 0.300 & 0.100 & - & - & - \\
\hline 21 & - & 1.000 & - & - & 1.000 & - & 1.000 & - & - & 0.706 & 0.294 & - & 0.692 & 0.039 & 0.269 & - & 0.500 & 0.059 & 0.118 & $0.029^{\circ}$ & 0.294 & - \\
\hline 22 & 0.075 & 0.900 & 0.025 & - & 1.000 & - & 1.000 & - & - & 0.775 & 0.225 & - & 0.825 & 0.025 & 0.150 & 0.075 & 0.475 & 0.100 & 0.175 & - & 0.125 & 0.050 \\
\hline 23 & - & 1.000 & - & - & 1.000 & - & 1.000 & - & - & 0.667 & 0.333 & - & 0.867 & 0.067 & 0.066 & - & 0.533 & 0.167 & 0.067 & - & 0.233 & - \\
\hline 24 & 0.105 & 0.895 & - & - & 1.000 & - & 1.000 & - & - & 0.700 & 0.300 & - & 0.895 & 0.026 & 0.079 & - & 0.974 & - & 0.026 & - & - & - \\
\hline 25 & 0.800 & 0.200 & - & - & 1.000 & - & 1.000 & - & - & 0.775 & 0.225 & - & 1.000 & - & - & 0.250 & 0.425 & 0.325 & - & - & - & - \\
\hline 26 & 0.800 & 0.200 & - & - & 1.000 & - & 1.000 & - & - & 0.750 & 0.250 & - & 1.000 & - & - & 0.075 & 0.650 & 0.225 & - & - & - & 0.050 \\
\hline 27 & 0.625 & 0.375 & - & - & 1.000 & 一 & 0.975 & 0.025 & - & 0.725 & 0.275 & - & 0.975 & 0.025 & - & 0.250 & 0.150 & 0.600 & - & - & - & - \\
\hline 28 & 1.000 & - & - & - & 0.975 & 0.025 & 1.000 & - & - & 0.550 & 0.450 & - & 0.950 & - & 0.050 & 0.175 & 0.100 & 0.725 & - & - & - & - \\
\hline 29 & 0.700 & 0.300 & - & - & 1.000 & - & 1.000 & - & - & 0.650 & 0.350 & - & 1.000 & - & - & 0.225 & - & 0.750 & - & 0.025 & - & - \\
\hline 30 & 0.775 & 0.225 & - & - & 0.975 & 0.025 & 1.000 & - & - & 0.850 & 0.150 & - & 0.950 & - & 0.050 & 0.225 & 0.575 & 0.075 & - & 0.025 & 0.075 & 0.025 \\
\hline 31 & 0.650 & 0.350 & - & - & 1.000 & - & 1.000 & - & - & 0.750 & 0.250 & - & 0.975 & - & 0.025 & 0.200 & 0.275 & 0.250 & 0.275 & - & - & - \\
\hline 32 & 0.400 & 0.600 & - & - & 1.000 & - & 1.000 & - & - & 0.775 & 0.225 & - & 1.000 & - & - & 0.300 & - & - & 0.700 & - & - & - \\
\hline 33 & 0.750 & 0.250 & - & - & 1.000 & - & 1.000 & - & - & 0.500 & 0.500 & - & 1.000 & - & - & - & - & - & 0.550 & 0.450 & - & - \\
\hline 34 & 0.538 & 0.462 & - & - & 1.000 & - & 1.000 & - & - & 0.500 & 0.500 & - & 0.769 & - & 0.231 & - & - & - & 1.000 & - & - & - \\
\hline 35 & 1.000 & - & - & - & 1.000 & - & 1.000 & - & - & 1.000 & - & - & 1.000 & - & - & - & 1.000 & - & - & - & - & - \\
\hline 36 & 1.000 & - & - & - & 1.000 & - & 1.000 & - & - & 1.000 & - & - & 1.000 & - & - & 0.167 & 0.792 & - & 0.041 & - & - & - \\
\hline 37 & 0.662 & 0.338 & - & - & 1.000 & - & 1.000 & - & - & 0.676 & 0.324 & - & 0.918 & 0.041 & 0.041 & 0.054 & 0.162 & 0.054 & 0.730 & - & - & - \\
\hline 38 & 0.900 & 0.100 & - & - & 1.000 & - & 0.975 & 0.025 & - & 0.600 & 0.400 & - & 1.000 & - & - & - & 0.025 & 0.100 & 0.825 & - & 0.025 & 0.025 \\
\hline 39 & 1.000 & - & - & - & 1.000 & - & 1.000 & - & - & 1.000 & - & - & 1.000 & - & - & 0.700 & 0.300 & - & - & - & - & - \\
\hline 40 & 1.000 & - & - & - & 1.000 & - & 1.000 & - & - & 1.000 & - & - & 1.000 & - & - & 0.300 & - & - & 0.700 & - & - & - \\
\hline 41 & 1.000 & - & - & - & 1.000 & - & 1.000 & - & - & 1.000 & - & - & 1.000 & - & - & 0.575 & 0.350 & - & 0.075 & - & - & - \\
\hline 42 & 0.708 & 0.208 & 0.084 & - & 1.000 & - & 1.000 & - & - & 1.000 & - & - & 1.000 & - & - & 0.500 & 0.167 & - & 0.167 & 0.166 & - & - \\
\hline 43 & 0.500 & 0.500 & - & - & 1.000 & - & 1.000 & - & - & 1.000 & - & - & 0.792 & - & 0.208 & 0.333 & 0.417 & 0.125 & 0.125 & - & - & - \\
\hline 44 & 0.933 & 0.067 & - & - & 1.000 & - & 1.000 & - & - & 0.367 & 0.633 & - & 0.700 & 0.100 & 0.200 & - & 0.500 & 0.200 & 0.233 & - & 0.067 & - \\
\hline
\end{tabular}


(sample 18) (Table 4). The mean proportion of polymorphic loci per population was $36.9 \pm 17.4 \%$ from the average of 44 sample lots and ranged from $8.3 \%$ (sample 12) to $75.0 \%$ (sample 18). Mean number of alleles per locus was $1.6 \pm 0.3$ in average and ranged from 1.2 (sample 35) to 2.3 (sample 22). The observed heterozygosity $\left(H_{0}\right)$ was $0.110 \pm 0.051$ in average and ranged from 0.025 (sample 8) to 0.225 (sample 17). The average heterozygosity $\left(H_{e}\right)$ was $0.131 \pm 0.053$ and ranged from 0.049 (sample 12) to 0.219 (sample 44).

Table 4 Genetic variabilities at 12 loci in 44 sample lots of the loach Misgurnus anguillicaudatus

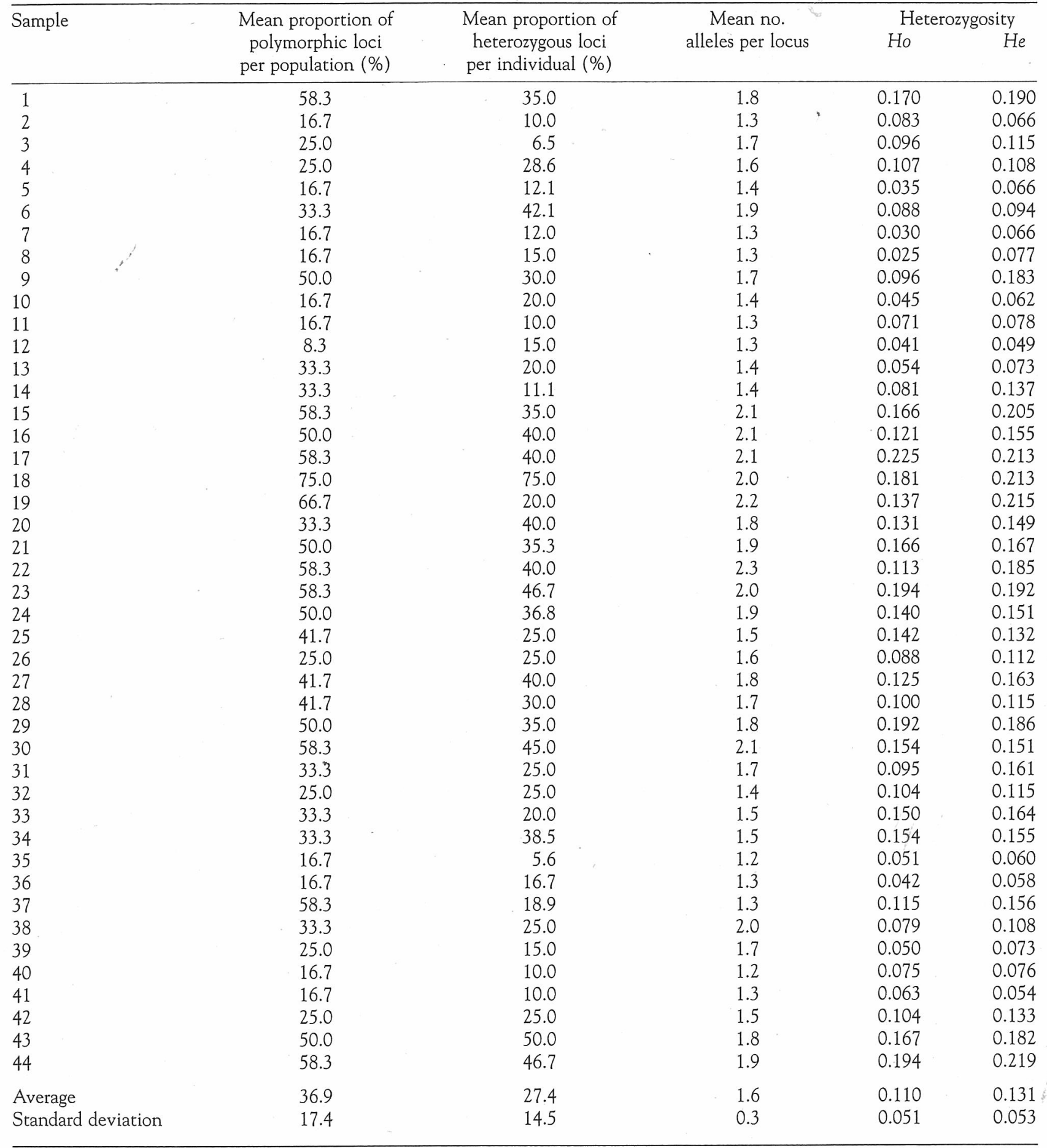




\section{Genetic differentiation}

The coefficient of gene differentiation $\left(G_{S T}\right)^{21}$ was estimated to be 0.774 , showing high genetic differentiation among populations. The genetic distance $(D)^{21}$ varied . from 0.001 to 0.399 . The minimum $D$-value $(0.001)$ was observed between Oono (sample 5) and Morioka (sample 7). The maximum value (0.399) was observed between Memanbetsu (sample 1) and Saito (sample 44). The average $D$-values between below-mentioned clustering groups and those within each group are shown in Table 5.

A dendrogram was drawn by the UPGMA method as shown in Fig. 2. When a vertical line was drawn across the dendrogram as an attempt to deliminate a group having $D=0.06$, six groups can be recognized (Fig. 2). The sampled localities of each group are shown in Fig. 3. Group 1 comprised only of the Memanbetsu population (sample 1$)$ was clearly separated from others $(D=0.286)$. Average genetic distances between group 1 and each of the other groups were large $(0.246-0.385)$ as seen in Table 5. Group 6 was then separated from other four groups at the D-value of 0.192 (Fig. 2). Average genetic distances between group 6 and each of the other four groups were relatively large $(0.216-0.089)$ as seen in Table 5. This group included loaches distributed in the central area of Honshu island from Shin-etsu, Kanto to Tokai districts, comprising Niigata (except for Hirokami population), Nagano, Tochigi, Saitama, Chiba, and Shizuoka Prefectures.(Fig. 3).

Table 5 Average $D$-values $( \pm S D)$ between groups and those within each group of the loach on the basis of 12 allozyme loci

\begin{tabular}{lccccc}
\hline & Group 2 & Group 3 & Group 4 & Group 5 & Group 6 \\
\hline Within group & $0.016 \pm 0.017$ & $0.027 \pm 0.012$ & $0.013 \pm 0.012$ & 0.041 & $(0.004-0.026)$ \\
& $(0.001-0.073)$ & $(0.009-0.038)$ & $(0.002-0.026)$ & $0.013 \pm 0.008$ \\
Group 1 & $0.285 \pm 0.050$ & $0.246 \pm 0.057$ & $0.371 \pm 0.007$ & $0.385 \pm 0.020$ & $0.258 \pm 0.021$ \\
& $(0.188-0.384)$ & $(0.181-0.324)$ & $(0.364-0.378)$ & $(0.371-0.399)$ & $(0.227-0.285)$ \\
Group 2 & & $0.069 \pm 0.027$ & $0.075 \pm 0.020$ & $0.122 \pm 0.030$ & $0.197 \pm 0.037$ \\
& & $(0.021-0.157)$ & $(0.044-0.121)$ & $(0.077-0.178)$ & $(0.116-0.292)$ \\
Group 3 & & $0.119 \pm 0.027$ & $0.148 \pm 0.030$ & $0.216 \pm 0.051$ \\
& & & $(0.075-0.169)$ & $(0.105-0.209)$ & $(0.104-0.303)$ \\
Group 4 & & & $0.065 \pm 0.019$ & $0.172 \pm 0.013$ \\
& & & & $(0.044-0.099)$ & $(0.141-0.191)$ \\
Group 5 & & & & $0.089 \pm 0.028$ \\
& & & & & $(0.048-0.134)$
\end{tabular}

\footnotetext{
${ }^{1}$ Group 1 consisted of a single sample lot (Memanbetu, Hokkaido). Groups 1-6 are as in the dendrogram shown in Fig. 2. Parentheses show range
} of $D$-value.

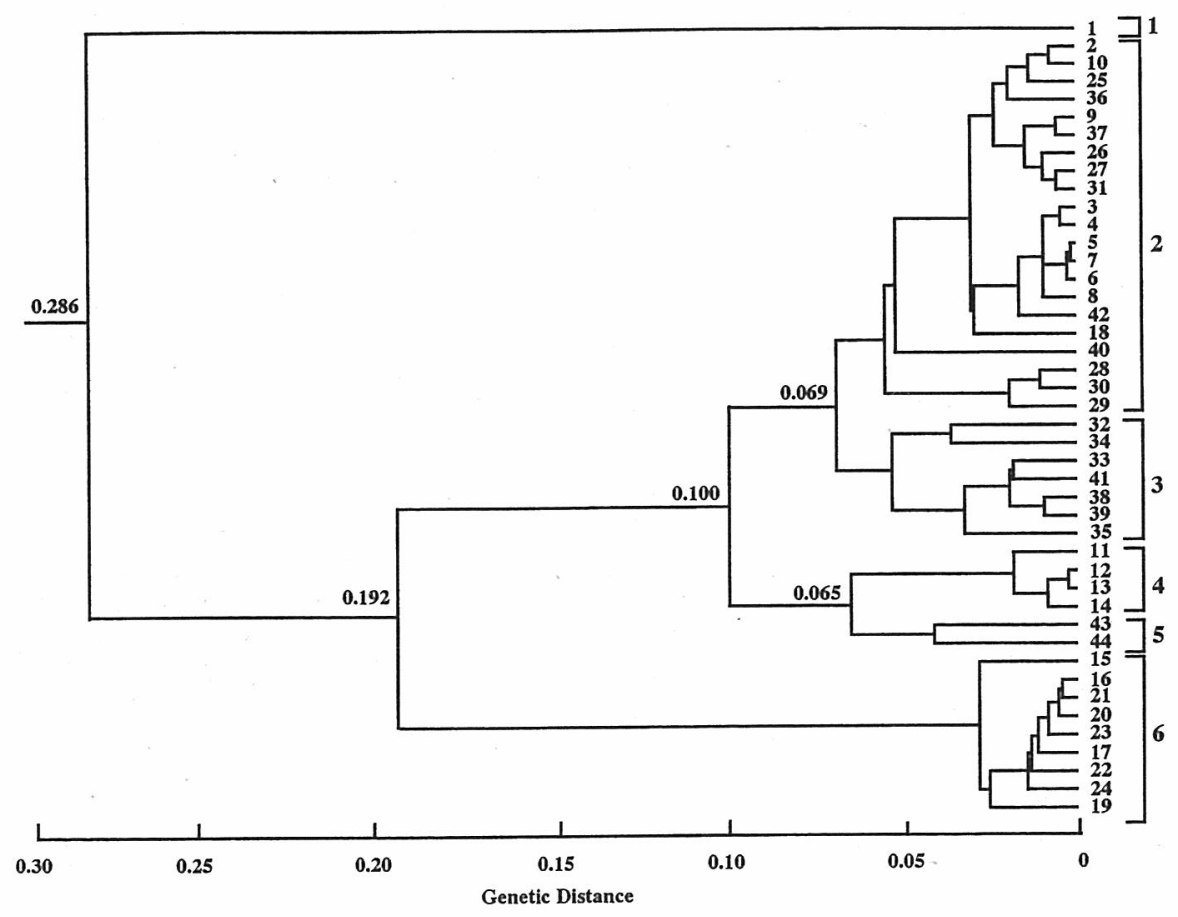

Fig. 2 UPGMA dendrogram for 44 sample lots of the loach Misgurnus anguillicaudatus. 
In other loaches from 34 localities, the cluster comprising groups 2 and 3 can be further separated from that comprising groups 4 and 5 at the $D=0.100$ (Fig. 2). The genetic distance between groups 2 and 3 was 0.069 and that between groups 4 and 5 was 0.065 (Table 5). Loaches of group 2 were discontinuously distributed in Japan (Fig. 3). Based on the geographical distribution pattern, they can be subgrouped into three: (i) Hokkaido-Northern Tohoku (Iwate and Akita Prefecture) district; (ii) Chubu (Gifu and Mie Prefectures)Kinki (Shiga, Fukui, and Kyoto Prefectures) district; and (iii) Sanyo (Hiroshima and Yamaguchi Prefectures)Shikoku (Ehime Prefecture) district. Only the Hirokami sample lot (18) was geographically isolated from the other subgroups. These subgroups cannot be classified by the genetic distances because geographically distant samples were often arranged as genetically close relationship as seen in Fig. 2.

Figure 3 also shows that loaches of group 3 distributed in Kinki (Kyoto Prefecture), San-in (Tottori and Shimane Prefectures), Sanyo (Hiroshima and Yamaguchi
Prefectures), and Shikoku (Ehime and Tokushima Prefectures) districts. Sample 32 was included with group 3 but sample 31 was collected very close to group 2: Similar situation was seen between samples 40 and 41 . Group 4 included samples from Southern Tohoku district comprising Yamagata, Miyagi, and Fukushima Prefectures. While genetically close, group 5 included loaches from Kochi Prefecture, Shikoku island and Miyazaki Prefecture, Kyushu island.

\section{DISCUSSION}

The proportion of polymorphic loci per population was 36.9 (mean) \pm 17.4 (SD)\% in the loach. This value is much higher than the reported average $(19.4 \pm 2.3 \%)$ based on allozyme studies in 41 marine teleost species. ${ }^{26}$ When compared with freshwater species, the value is relatively similar to that for fluvial dace $(22 \%)^{27}$ but higher than fluvial sculpin (3\%), ${ }^{28}$ and land-locked stickleback (4\%). ${ }^{29}$ Thus, genetic polymorphism is maintained in the
Fig. 3 Geographical distribution of six genetically distinct groups, including 44 sample lots of the loach Misgurnus anguillicaudatus.

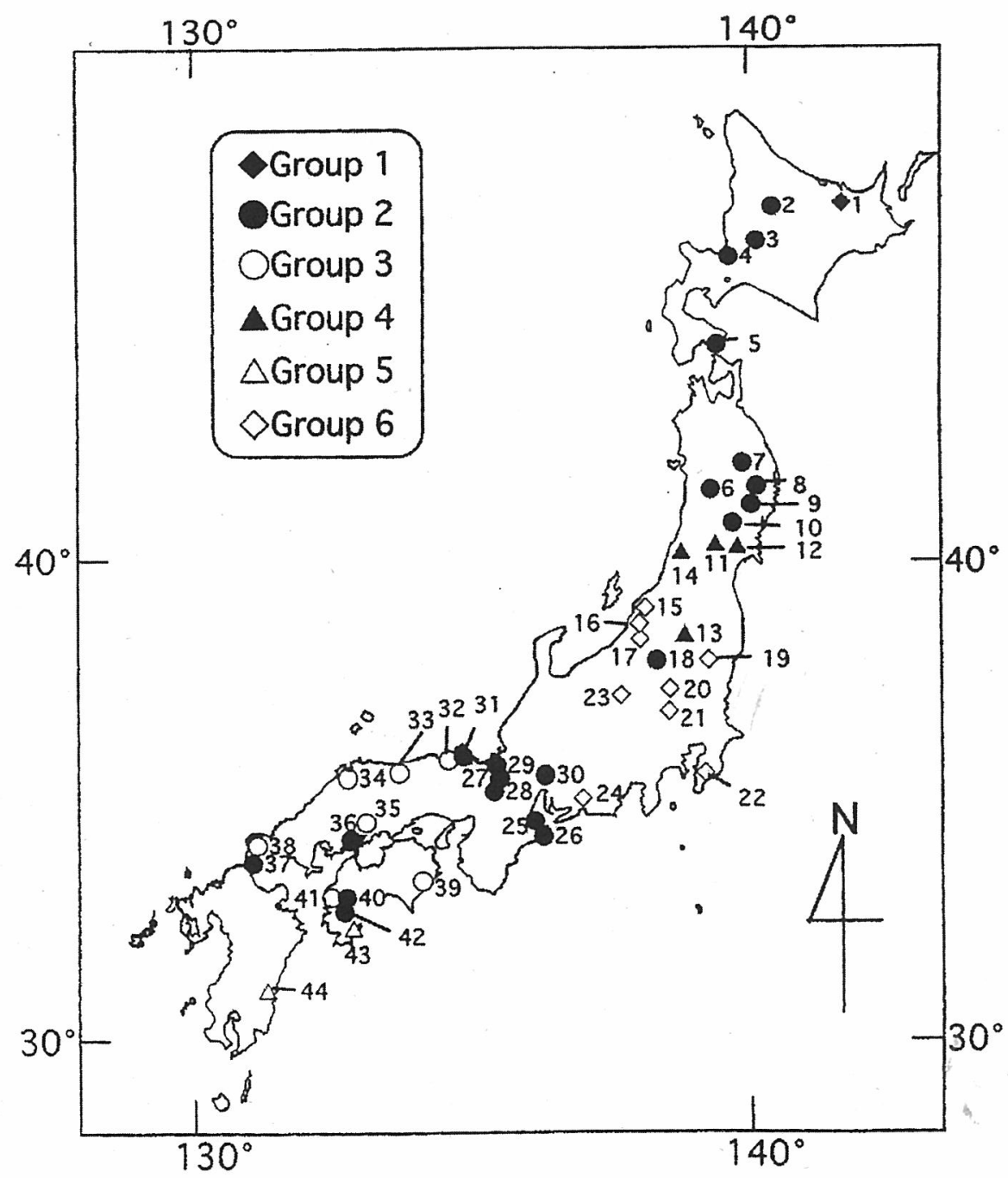


loach. This conclusion is also suggested by the mean proportion of heterozygous loci $(27.4 \pm 14.5 \%)$ and the mean number of alleles per locus $(1.6 \pm 0.3)$ in the loach.

The average heterozygosity $(\mathrm{Ho}=0.110 \pm 0.051 ; \mathrm{He}=$ $0.131 \pm 0.053$ ) estimated in the loach is unusually higher than the average values $(0.059+0.007)$ from 41 marine fishes, ${ }^{24}$ and the values reported in fluvial or land-locked species such as dace (0.04), ${ }^{27}$ sculpin (0.01), ${ }^{28}$ stickleback $(0.01),{ }^{29}$ freshwater goby $(0.04),{ }^{30}$ medaka (0.004$0.111){ }^{31}$ Thus, the loach is maintaining much higher heterozygosity than other fluvial fishes.

The $G_{S T}$ value (0.774) of the total population is nearly equivalent to those estimated in other Japanese fluvial fishes such as freshwater goby $(0.698)^{30}$ and southern group of brook lamprey (0.629). ${ }^{32}$ This suggests a large proportion of genetic variation in the loach which may be due to differences among populations from different localities.

Loaches can be grouped into six as shown in the dendrogram. Group 1 (Memanbetsu, Hokkaido) was a highly divergent population which was separated from others at $D=0.286$. Genetic differences between Memanbetsu population (group 1) and other populations of groups 2-6 were in the range of $D$-values approximately between 0.4 and 0.2. The group 6 was separated from others at $D=0.192$. Average genetic distances between group 6 and others were in the range approximately between 0.2 and 0.1 . These $D$-values are lower than those between different species of freshwater teleosts in the same genus reported in Lepomis $(D=0.627),{ }^{33,34}$ Menidia $(D=0.421),{ }^{35}$ Oncorhynchus $(D=0.392),{ }^{36}$ Salmo $(D=0.496),{ }^{36}$ Salvelinus $(D=1.169),{ }^{36}$ Zacco $\quad(D=$ $0.640),{ }^{37}$ and Rhinogobius $(D=0.35-1.77),{ }^{38}$ but nearly equivalent to those between subspecies in the same species reported in ayu ${ }^{39}(D=0.242)$. Shimizu et al. ${ }^{30}$ reported that $D$-values among the five groups of Rhinogobius ranged from 0.07 to 0.23 and suggested that the highest genetic differentiation among them was the species or subspecies level. Nei found that in a variety of animals, $D$ is approximately 1.0 for between-species comparisons, around 0.1 for subspecies, and 0.01 for local races. ${ }^{24}$ Ayala reported that the $D$-value between subspecies is approximately $0.20 . .^{40}$ Judging from the above criteria, genetic differentiation among group 1, group 6 , and others (groups 2-5) of the Japanese diploid loach is likely to be inter-subspecies level. Genetically divergent groups 1 and 6 are geographically separated from others and distribute in the northern Hokkaido and the central area of Honshu Island, respectively.

In the near future, mechanisms of reproductive isolation among them should be elucidated by experimental hybridizations between them and subsequent observations of survival and reproductive potential of the resultant hybrids. In the case of Japanese frog species, Rana japonicus, distinct genetic differentiation was observed between the eastern and western geographical groups at the mean $D$-value of $0.156 .^{41}$ In reciprocal hybrids between these two races, a remarkable preponderance of males was observed. ${ }^{42}$ In interspecific hybridization of medaka species, female hybrids produced unreduced diploid eggs, while male hybrids were sterile. ${ }^{12}$ In the loach, Zhang and Arai have found relatively high frequencies of natural triploids in Ichinomiya town, Aichi Prefecture and Hirokami village, Niigata Prefecture. ${ }^{10}$ Relatively high frequency of natural triploids was also observed in Memanbetsu, Hokkaido (S. Horie and K. Arai, unpubl. data, 1999). These localities seem to be in zones between genetically distinct groups shown in the present study. An accidental hybridization between genetically distinct loaches may give rise to the occurrence of sterile progeny or semi-fertile progeny which are able to produce unreduced eggs. In Hirokami, the occurrence of unreduced diploid eggs was found in diploid loach. ${ }^{10}$

In other populations, the cluster including groups 2 and 3 was separated from that including groups 4 and 5 at the $D$-value of 0.100 . The loaches of group 2 distributed all over Japan except for the central part (Shin-etsu, Kanto, and Tokai district) and Kyushu district. Group 3 was genetically close to group 2 and its distribution overlapped with group 2 in Chugoku and Shikoku district. Some samples, such as 31 and 32 , and 40 and 41 , were geographically very close, but they were separated into different groups (2 and 3). Group 4, distributed in Southern Tohoku district, was genetically close to group 5, distributed in Shikoku and Kyushu district. Genetic differentiation among these populations seems to be a local race level, judging from the aforementioned examples. $^{24}$

Although group 2 showed discontinuous distribution, these samples are likely to be categorized into three geographically independent subgroups: Hokkaido-Northern Tohoku, Chubu-Kinki and Western Chugoku-Shikoku, with the exception of Hirokami samples in Niigata Prefecture. These populations were not clearly separated as independent clusters by the present genetic studies using 12 allozyme loci. The analyses suggest that the loach has a tendency to split to a number of local populations, although the question of whether each population was wild or a mixture due to artificial factors such as implantation remains. Observed inconsistencies between genetic and geographical distances in several populations might be due to an insufficient number of samples and genetic loci examined. These problems must be clarified by a series of further studies on more samples using additional gene markers such as mtDNA and microsatellites.

\section{ACKNOWLEDGMENTS}

We wish to thank Professor H. Nakagawa, Hiroshima University for his expert guidance, valuable suggestions, and encouragement throughout this study. We are very grateful to the following persons who helped to collect 
fish specimens: Dr A. Suzuki (Tokyo University of Agriculture), Mr K. Suzuki (Hokkaido Fish Hatchery), Mr I. Matsuoka, Mr T. Suganuma (Higashikawa Town), Dr E. Yamaha (Hokkaido University), Mr Y. Ito (Morioka Daiyon High School), Professor H. Ida, Dr T. Asahida (Kitasato University), Mr S. Yogo (Yamagata Prefectural Fisheries Experimental Station), Mr M. Kawamura (Aizu Wakamatsu City), Professor A. Kijima (Tohoku University), Mr N. Tomioka, Mr Y. Ishikawa, M. Sato (Niigata Prefectural Inland Fisheries Experimental Station), Professor S. Hamaguchi, Professor M. Sakaizumi (Niigata University), Mr I. Sato, Mr K. Watanabe (Niigata Prefectural Maritime High School), Dr T. Ueda (Utsunomia University), Mr Y. Shou (Seibutsukagaku Kenkyusho), $\mathrm{Dr}$ H. Hosoya (National Research Institute of Fisheries Science), Professor H. Kurokura (The University of Tokyo), Dr Y. Fujioka (Shiga Prefectural Fisheries Experimental Station), Dr T. Kobayashi (Kinki University), Dr I. Nakayama (National Research Institute of Aquaculture), Mr M. Tanaka (Mineyama T.), Professor M. Nishida (The University of Tokyo, Ocean Research Institute), Dr E. Yamamoto (Tottori Prefectural Fisheries Experimental Station), Dr Y. Jou (Tokushima Prefectural Fisheries Experimental Station), Mr H. Yamashita (Ehime Prefectural Fisheries Experimental Station), Mr M. Yamaki (Ehime Prefectural Uwajima Fisheries High School), Mr M. Okabe (Kochi Prefectural Fisheries Experimental Station), Dr H. Sakai (National Fish University), and the late Dr M. Akasaki (Professor Emeritus, Miyazaki University). We are also grateful to $\mathrm{Dr}$ T. Umino, Dr Q. Zhang, Mr K. Morishima, Mr T. Hashimoto, Mr K. Taniura, Mr S. Momotani, and Mr M. Hayashi (Hiroshima University) for their technical help. We also thank anonymous reviewers for their valuable comments.

\section{REFERENCES}

1. Saitoh K. Asian pond loach. In: Kawanabe H, Mizuno N (eds). Freshwater Fishes of Japan. Yamatokeikokusha, Tokyo, 1989; 382 385 (in Japanese).

2. Oliva O, Hensel K. Some remarks on eastern Asiatic loaches of the genus Misgurnus (Cobitidae). Jpn. J. Ichthyol. 1961; 8: 86-91.

3. Li K, Li Y, Zhou D. A comparative study of the karyotypes in two species of mud loaches. Zool. Res. 1983; 4: 75-81.

4. Hitotsumachi S, Sasaki M, Ojima Y. A comparative karyotype study in several species of Japanese loaches (Pisces, Cobitidae). Jpn. J. Genet. 1969; 44: 157-161.

5. Ojima Y, Takai A. The occurrence of spontaneous polyploid in the Japanese common loach, Misgurnus anguillicaudatus. Proc. Japan Acad. 1979; 55: 487-491.

6. Arai K, Matsubara K, Suzuki R. Karyotype and erythrocyte size of spontaneous tetraploidy and triploidy in the loach Misgurnus anguillicaudatus. Nippon Suisan Gakkaishi 1991; 57: 2167-2172.

7. Arai K, Mukaino M. Electrophoretic analysis of the diploid progenies from triploid $\mathrm{x}$ diploid crosses in the loach Misgurnus anguillicaudatus (Pisces: Cobitidae). J. Exp. Zool. 1998; 280: 368-374.
8. Kimura M. Protein polymorphism and geographic variation in the loach Misgurnus anguillicaudatus. Anim. Blood Grps. Biochem. Genet. 1978; 9: 13-20.

9. Dong S, Taniguchi N, Ishida R. Two types of the loach Misgurnus anguillicaudatus collected from Torentsu River. Jpn. J. Ichthyol. 1999; 46: 83-90.

10. Zhang $\mathrm{Q}$, Arai K. Distribution and reproductive capacity of natural triploid individuals and occurrence of unreduced eggs as a cause of polyploidization in the loach Misgurnus anguillicaudatus. Icthyol. Res. 1999; 46: 153-161.

11. Dawley RM. An introduction to unisexual vertebrates. In: Dawley RM, Bogart JP (eds). Evolution and Ecology of Unisexual Vertebrates. Bulletin 466, New York State Museum, Albany, 1989; $1-18$.

12. Sakaizumi M, Shimizu Y, Matsuzaki T, Hamaguchi S. Unreduced diploid eggs produced by interspecific hybrids between Oryzias latipes and O. curvinotus. J.' Exp. Zool. 1993; 266: 312-318.

13. Zhang Q, Arai K. Flow cytometry for DNA contents of somatic cells and spermatozoa in the progeny of natural tetraploid loach. Fisheries Sci, 1996; 62: 870-877.

14. Clayton JW, Tretiak DN. Amine-citrate buffers for $\mathrm{pH}$ control in starch gel electrophoresis. J. Fish. Res. Bd. Canada 1972; 29: 1169-1172.

15. Ridgway GJ, Sherburne SW, Lewis RD. Polymorphisms in the esterases of Atlantic herring. Trans. Am. Fish. Soc. 1970; 99: 147-151.

16. Allendorf FW, Mitchell N, Ryman N, Stahl G. Isozyme loci in brown trout (Salmo trutta L.): detection and interpretation for population data. Hereditas 1977; 86: 179-190.

17. Aebersold PB, Winans GA, Teel DJ, Milner GB, Utter FM. Manual for starch gel electrophoresis: a manual for the detection of genetic variation. NOAA Technical Report NMFS 1987; 61: 1-19.

18. Shaklee JB, Allendorf FW, Morizot DC, Whitt GS. Gene nomenclature for protein-coding loci in fish. Trans. Am. Fish. Soc. 1990; 119: 2-15.

19. Lewontin RC, Hubby JL. A molecular approach to the study of genetic heterozygosity in natural populations. II. Amount of variation and degree of heterozygosity in natural populations of Drosophila pseudoobscura. Genetics 1966; 54: 595-609.

20. Nei M, Roychoudhury AK. Probability of fixation and mean fixation time of an over dominant mutation. Genetics 1973; 74: 371-380.

21. Nei M. Genetic distance between populations. Am. Nat. 1972; 106: 283-292.

22. Swofford DL, Selander RB. BIOSYS-1: A FORTRAN program for the comprehensive analysis of electrophoretic data in population genetics and systematics. J. Hered. 1981; 72: 281-283.

23. Sneath PHA, Sokal RR. Numerical Taxonomy. W. H. Freedom and Co., San Francisco, 1973.

24. Nei M. Molecular Population Genetics and Evolution. NorthHolland, Amsterdam, 1975.

25. Nei M. Molecular Evolutionary Genetics. Columbia University Press, New York, 1987.

26. Fujio Y, Kato Y. Genetic variation in fish populations. Nippon Suisan Gakkaishi 1979; 45: 1169-1178.

27. Hanzawa H, Taniguchi N, Numachi K. Geographical differentiation in populations of Japanese dace Triborodon hakonensis deduced from allozyme variation. Zool. Sci. 1988; 5: 449-461.

28. Goto A, Andoh T. Genetic divergence between the sibling species of river-sculpins, Cottus amblystomopsis and C. nozawae, with special reference to speciation. Env. Biol. Fish. 1990; 28: 257-266. 
29. Taniguchi N, Honma Y, Kawamata K. Genetic differentiation of freshwater and anadromous three spine sticklebacks (Gasterosteus aculeatus) from northern Japan. Jpn. J. Ichthyol. 1990; 37: 230238.

30. Shimizu T, Taniguchi N, Mizuno N. An electrophoretic study of genetic differentiation of a Japanese freshwater goby, Rhinigobius flumineus. Jpn. J. Ichthyol. 1993; 39: 329-343.

31. Takata K, Hoshino M, Magtoon W, Nadee N, Uwa H. Genetic differentiation of Oryzias minutillus in Thailand. Jpn. J. Ichthyol. 1993; 39: 319-328.

32. Yamazaki Y, Goto A. Genetic differentiation of Lethenteron reissneri populations, with reference to the existence of discrete taxonomic entities. Icthyol. Res. 1996; 43: 283-299.

33. Avice JC, Smith MS. Biochemical genetics of sunfish. I. Geographic variation and subspecific intergradation in the bluegill, Lepomis machrochirus. Evolution 1974; 28: 42-56.

34. Avice JC, Smith MS. Biochemical genetics of sunfish. II. Genetic similarity between hybridizing species. Am. Nat. 1974; 108: $458-472$.

35. Johnson MS. Biochemical systematics of the atherinid genus Menidia. Copeia 1975; 662-691.
36. Numachi K. Fish population genetics by isozyme markers. Tansuigyo (Freshwater Fish) 1982; 8: 41-57 (in Japanese).

37. Okazaki T, Watanabe M, Mizuguchi K, Hosoya K. Genetic differentiation between two types of dark chub, Zacco temmincki, in Japan. Jpn. J. Ichthyol. 1991; 38: 133-140.

38. Masuda Y, Ozawa T, Enami S. Genetic differentiation among eight color types of the freshwater goby, Rhinogobius brunneus, from Western Japan. Jpn. J. Ichthyol. 1989; 36: 30-41.

39. Sawashi Y, Fujimito H, Azuma M, Nishijima S, Nishida M. Genetic and morphological characteristics and distribution of the Ayu Plecoglossus altivelis in the northern Ryukyus. Nippon Suisan Gakkaishi 1993; 59: 191-199.

40. Ayala FJ. Genetic differentiation during the speciation process. Evol. Biol. 1975; 8: 1-78.

41. Sumida M, Nishioka M. Genetic differentiation of the Japanese brown frog, Rana japonicus, elucidated by electrophoretic analyses of enzymes and blood proteins. Sci. Rep. Lab. Amphibian Biol. Hiroshima Univ. 1994; 13: 137-171.

42. Sumida M. Incipient intraspecific isolating mechanisms in the Japanese brown frog Rana japonicus. J. Herpatol. 1996; 30: 333-345. 